\title{
Envelope Domain Formulation for the Analysis of the Nonlinear Transient Dynamics of Coupled Oscillators
}

\author{
Sergio Sancho, Member, IEEE, Almudena Suarez, Fellow, IEEE, Franco Ramirez, Senior Member, IEEE
}

\begin{abstract}
A reduced-order envelope-domain formulation of coupled-oscillator systems based on realistic nonlinear models of the oscillator elements is presented for the first time to our knowledge. The formulation, based on numerical models of the transistor-based oscillators, enables an accurate prediction of the nonlinear dynamics of the coupled system, including the oscillation build-up, the locked and unlocked states, and the oscillator on-off switching. To increase the applicability of the method, both admittance- and impedance-type models are extracted through harmonic balance simulations, under a voltage and current excitation, respectively, at the node/branch where the oscillator is connected to the coupled system. They are used to derive a nonlinear differential-equation system able to describe the transient dynamics of the entire structure. Because the oscillators are coupled through current injection, the impedance-based formulation is formally different from the admittance one, so it requires a dedicated derivation. For illustration, the method has been applied to exhaustively investigate the nonlinear dynamics of a system of three FET-based oscillators at $5 \mathrm{GHz}$. Very good agreement has been obtained with both circuit-level envelope transient (when applicable) and with measurements.
\end{abstract}

Index Terms - Coupled-oscillator systems, transient analysis

\section{INTRODUCTION}

$\mathrm{C}$ OUPLED-OSCILLATOR systems are used in a variety of applications, including power combination, beam steering, wireless distribution of synchronization signals and sensors [1]-[12]. However, the simulation of these systems is demanding. Time-domain analyses might not be applicable at microwave frequencies and harmonic balance (HB) requires the individual fulfillment of the oscillation condition in each oscillator circuit. This can be achieved by introducing one auxiliary generator (AG) [13], [14] in each oscillator element, so, in a system of $M$ coupled oscillators, $M$ oscillation conditions must be satisfied [15], given by the zero value of the current-to-voltage ratio at each of the introduced AGs, $Y_{A G, i}=$ 0 , where $i=1$ to $M$. In commercial software, this is solved through the simultaneous optimization of the AG amplitudes, phase shift, frequency and analysis parameter(s). However, and due to the lack of symmetry of the coupled system, convergence failures will arise from a certain number of oscillator elements [16]. On the other hand, the circuit-level envelope-transient

\footnotetext{
This paper is an expanded version from the IEEE MTT-S International Microwave Symposium (IMS 2020), Los Angeles, CA, USA, August 4-6, 2020. This work was submitted for review on July, 21-th, 2020. This work was supported by the Spanish Ministry of Economy and Competitiveness and the
}

formulation [17]-[19] requires a proper initialization of each of the $M$ oscillator elements, and, in the presence of a high number $M$ of elements, convergence problems may be encountered when one or more of these elements undergo strong amplitude and frequency variations, for instance, under unlocked conditions. In order to cope with the mentioned analysis problems, several previous works [15], [16], [20] propose the use of oscillator models extracted from a HB simulation of the standalone oscillator circuit. These models are based on the linearization of the admittance function $Y$ of each oscillator about its standalone free-running oscillation, fulfilling $Y\left(V_{o}, \omega_{o}\right)=0$. The linearization is carried out obtaining the free-running oscillation with the aid of an AG and then applying finite differences [13] to its amplitude and frequency about the free-running point.

The above semi-analytical method has the advantage of enabling an efficient and realistic prediction of the behavior of practical systems of transistor-based oscillators. However, because the HB models are extracted linearizing the oscillator circuits about their free-running solutions (usually assumed equal), only periodic and modulated states with small amplitude and frequency deviations with respect to their original freerunning points can be simulated. Thus, the linearization severely limits the scope of application of the semi-analytical method, since it is unable to cover the oscillation build-up, as well as unlocked regimes or the behavior under the oscillator switching. Although the full nonlinear dynamics can be addressed with reduced-order models of the Van der Pol type [21], the accuracy of these models is usually insufficient when dealing with transistor-based oscillators, containing several nonlinearities and parasitic elements.

The purpose of this work is to develop a realistic envelopedomain formulation of coupled-oscillator systems, based on nonlinear reduced-order models of the oscillator elements. Unlike the linearization performed in [15], [16], [22], each oscillator model relies on a nonlinear admittance function, $Y(V, \omega)$, that is extracted through a double sweep in the excitation amplitude $V$ and frequency $\omega$ of an AG, performing a HB simulation at each sweep step. The resulting set of bivariate nonlinear-admittance functions $Y_{i}\left(V_{i}, \omega_{i}\right)$ will be used to develop an envelope-domain formulation of the coupled system. This will be obtained by associating a time-

European Regional Development Fund (ERDF/FEDER) under the research project TEC2017-88242-C3-1-R. The authors are with Dpto. Ingeniería de Comunicaciones, Universidad de Cantabria., ETSIIT, Av. Los Castros 39005, Santander, Spain (e-mail: sanchosm@unican.es) 
differentiation operator to the frequency dependence, as done in the circuit-level piecewise envelope transient [17]-[19], [23]. This work expands [24], with a generalization of the oscillator modeling technique and a detailed exploration of the nonlinear dynamics of the coupled system. The generalized method should be applicable to transistor-based oscillators of arbitrary topology, and the reduced-order envelope-domain system should be able to predict both locked and unlocked states, as well as the build-up transients. This realistic and efficient analysis of the nonlinear dynamics of the coupled system is presented here for the first time, to the best of our knowledge.

As stated, the nonlinear oscillator models are extracted from circuit-level $\mathrm{HB}$, and, as any other $\mathrm{HB}$ simulation, the procedure may be subject to convergence problems. Most often, these problems come from the singularity of the Jacobian matrix of the HB system during the Newton iteration [19], [25]. As is well known in nonlinear-circuit analysis [26], [27], the singularities can be circumvented through a continuation method [26]-[28]. One of the most commonly used is the parameter switching [26], [27], based on switching the actual analysis parameter to a fast-varying state variable, and transforming the physical parameter into an unknown to be determined. In our model extraction, a conceptually analogous procedure would be to change the independent excitation from voltage to current. The current excitation leads to a nonlinear model of impedance type, depending on the excitation frequency and the current amplitude. However, the oscillators are coupled through current injection, so the impedance-based formulation requires a dedicated derivation, here presented for the first time. The possibility to use both admittance and impedance models generalizes the method and significantly increases its applicability. For illustration, the method has been used to exhaustively investigate the nonlinear dynamics of a system of three FET-based oscillators at $5 \mathrm{GHz}$. Very good agreement has been obtained with both circuit-level envelope transient (when applicable) and with measurements.

The paper is organized as follows. Section II presents the coupled-system analysis based on admittance models. Section III describes the analysis based on impedance models. Section IV presents a detailed investigation of the dynamics of a system of coupled transistor-based oscillators.

\section{ENVELOPE-DOMAIN ANALYSIS BASED ON A NONLINEAR- ADMITTANCE FORMULATION}

\section{A. System formulation}

The HB system governing the behavior of each free-running oscillator is written as:

$$
\begin{aligned}
& I(\bar{A}, V, \phi, \omega)=0, \\
& \bar{H}(\bar{A}, V, \phi, \omega)=0
\end{aligned}
$$

Equation (1a) is the first harmonic equation of the Kirchhoff current law (KCL) at a particular observation node $q$, and system (1b) contains the rest of the HB equations. The variables $(V, \phi)$ agree with the amplitude and phase of the first harmonic component of the voltage signal at the node $q$, whereas the vector $\bar{A}$ contains the rest of the harmonics of the state variables and $\omega$ is the free-running oscillation frequency. Applying the implicit function theorem [13], the components in $\bar{A}$ can be derived from equation (1b) in terms of $(V, \phi, \omega)$ and then introduced in (1a), obtaining $I(V, \omega)=Y(V, \omega) V e^{j \phi}=0$, where $Y(V, \omega)$ is the admittance function at $q$, calculated at the first harmonic. In practice, this function will be obtained connecting an AG in parallel at the node $q$ [Fig. 1(a)]. Then, a double sweep is carried out in the AG amplitude $V$ and frequency $\omega$, performing a HB simulation at each sweep step [29], [30]. The admittance function is calculated as:

$$
Y(V, \omega)=\frac{I_{1}\left(V_{A G}=V, \omega_{A G}=\omega\right)}{V}
$$

where $I_{1}$ is the first harmonic component of the current entering the node $q$ from the AG. Now the case of a system of $M$ coupled oscillators, such as the one shown in Fig. 1(b), will be considered. The oscillator components are assumed different, so a function $Y_{i}\left(V_{i}, \omega\right)$ will be derived for each $i$-th oscillator element. In each case, the observation node $q_{i}$ corresponds to the one at which the oscillator is connected to the coupling network. The schematic of the admittance-based system is shown in Fig. 1(b).

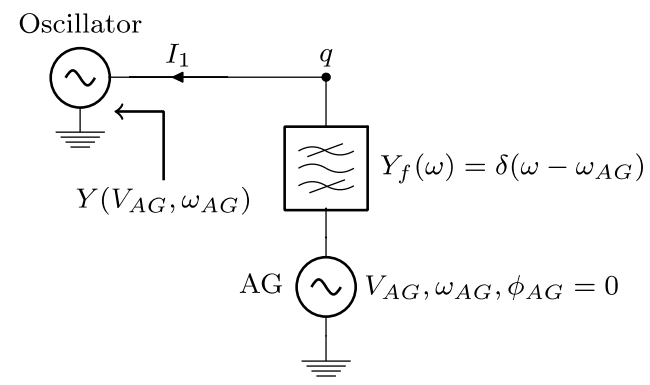

(a)

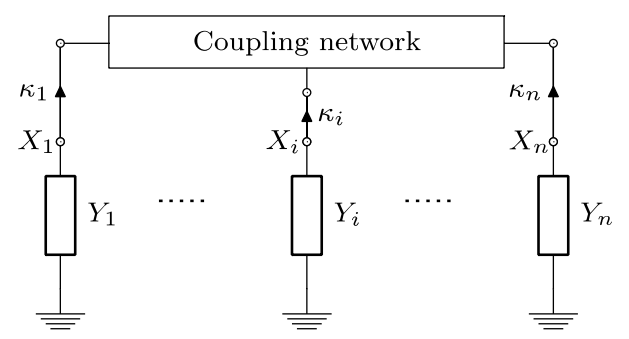

(b)

Fig. 1. (a) Schematic of the circuit used to calculate the admittance function $Y(V, \omega)$. (b) Schematic of the system of coupled oscillators indicating the variables used in the admittance-based formulation.

To derive the differential-equation system, one will initially consider the steady-state system in a locked condition at the fundamental frequency $\omega$ :

$$
Y_{i}\left(V_{i}, \omega\right) X_{i}+\kappa_{i}=0, \quad \kappa_{i}=\sum_{k=1}^{M} Y_{i k}^{c}(\omega) X_{k}=0
$$

where $i=1, \ldots, M$. In (3), each $X_{i} \equiv V_{i} e^{j \phi_{i}}$ is the first harmonic of the voltage signal of each $i$-th oscillator at the node $q_{i}$, and $\kappa_{i}$ is the first harmonic of the current entering the coupling network. The current phasors $\kappa_{i}$ have been expressed in terms of the admittance matrix of the linear coupling network, whose components are $Y_{i k}^{c}(\omega)$. System (3) will be fulfilled in locked conditions at the frequency $\omega=\omega_{s}$ for the values $\left(V_{i}, \phi_{i}, \omega_{s}\right)$ which, in general, will be different from 
those of the individual free-running oscillators (when isolated from the system).

Now the transient of the coupled-oscillator system will be addressed. During this transient the amplitude and phase of each oscillator are time varying. The corresponding system is derived from (3) by modifying the frequency variable in the form $\omega \rightarrow \omega_{o}+D_{t} / j$ [23], where $D_{t}$ is the time-derivative operator and $\omega_{o}$ is the mean value of the free-running frequencies of the oscillators in the array, which is arbitrarily taken as fundamental frequency of the Fourier basis. Thus, we obtain the following system of nonlinear differential equations:

$$
\begin{aligned}
& Y_{i}\left(V_{i}(t), \omega_{o}+\frac{D_{t}}{j}\right) X_{i}(t)+\sum_{k=1}^{n} Y_{i k}^{c}\left(\omega_{o}+\frac{D_{t}}{j}\right) X_{k}(t)=0, \\
& i=1, \ldots, M
\end{aligned}
$$

We emphasize that, unlike the analyses [15], [16], [20], the nonlinear admittance functions $Y_{i}\left(V_{i}, \omega\right)$ in (4) are not linearized about the locked steady state. On the contrary, the nonlinear dependence of these functions on the amplitude variable will be used to predict the full transient dynamics.

During the transient state, the time variation of the phasors $X_{i}(t)$ is in general much slower than the time scale associated with the oscillation frequency $\omega_{o}$. Thus, these components are narrowband signals that can be expressed as [17]:

$$
X_{i}(t)=\int_{-\omega_{b}}^{\omega_{b}} X_{i}(\omega) e^{j \omega t} d \omega, \quad i=1, \ldots, M
$$

where $\omega_{b}$ is the maximum bandwidth of the time-varying phasors. Using this result, the dependence of the admittance functions on the complex frequency can be approached by a first-order Taylor series, providing:

$$
a_{0}^{i}\left(V_{i}\right) X_{i}(t)+a_{1}^{i}\left(V_{i}\right) \dot{X}_{i}(t)+\sum_{k=1}^{n} Y_{i k}^{c}\left(\omega_{o}\right) X_{k}(t)=0
$$

where:

$$
a_{0}^{i}\left(V_{i}\right) \equiv Y_{i}\left(V_{i}, \omega_{o}\right), \quad a_{1}^{i}\left(V_{i}\right) \equiv-j \frac{\partial Y_{i}\left(V_{i}, \omega_{o}\right)}{\partial \omega}
$$

for $i=1, \ldots, M$. Note that once the nonlinear admittance functions $a_{0}^{i}\left(V_{i}\right)$ are available, the derivatives $a_{1}^{i}\left(V_{i}\right)$ are extracted through finite differences in a straight-forward manner [30]. On the other hand, the frequency dependence of the linear coupling-network admittance matrix has been neglected, since its components remain nearly constant in the frequency range $\left[\omega_{o}-\omega_{b}, \omega_{o}+\omega_{b}\right]$. System (6) provides for the first time to our knowledge a set of nonlinear ODEs describing the transient behavior of a multi-oscillator system. In order to better understand the system dynamics, it is more illustrative to express the equation of each oscillator of (6) in terms of the phase and amplitude variables:

$$
\begin{aligned}
& a_{0}^{i}\left(V_{i}\right) V_{i}(t)+a_{1}^{i}\left(V_{i}\right)\left(\dot{V}_{i}(t)+j V_{i}(t) \dot{\phi}_{i}(t)\right)+ \\
& +\sum_{k=1}^{n} Y_{i k}^{c}\left(\omega_{o}\right) V_{k}(t) e^{j\left(\phi_{k}(t)-\phi_{i}(t)\right)}=0
\end{aligned}
$$

for $i=1, \ldots, M$. Each equation depends nonlinearly on the voltage amplitudes and phases through the functions $a_{0,1}^{i}\left(V_{i}\right)$ and the exponential components, respectively. Splitting each complex equation in (8) into real and imaginary parts, the whole system can be rewritten in compact form as:

$$
\dot{\bar{y}}(t)=\bar{f}(\bar{V}(t), \bar{\phi}(t)), \quad \bar{y}=\left(\begin{array}{l}
\bar{V} \\
\bar{\phi}
\end{array}\right)
$$

where the set of state variables is composed of the components in the vectors $\bar{V}=\left(V_{1}, \ldots, V_{M}\right)^{t}$ and $\bar{\phi}=\left(\phi_{1}, \ldots, \phi_{M}\right)^{t}$. Expressing the first harmonic in terms of the phase and amplitude variables as $V_{i}(t) e^{j\left(\omega_{o} t+\phi_{i}(t)\right)}$, it is seen that the instantaneous frequency of each $i$-th oscillator is $\omega_{o}+\dot{\phi}_{i}(t)$.

The procedure to obtain system (9) is summarized as:

1) Obtain the nonlinear admittance functions $Y_{i}\left(V_{i}, \omega\right)$ of the whole set of oscillators $(i=1, \ldots, M)$ in circuit-level HB using the AG technique [29], [30] and build the functions $a_{0}^{i}\left(V_{i}\right)$ and $a_{1}^{i}\left(V_{i}\right)$.

2) Build the nonlinear system of ODEs (9) from these functions and use it to simulate the transient trajectories of the amplitude and phase of each oscillator of the array through timeintegration.

From (8), it can be straightforwardly derived that:

$$
\bar{f}\left(\bar{V}(t), \bar{\phi}_{\alpha}(t)\right)=\bar{f}(\bar{V}(t), \bar{\phi}(t)), \quad \forall \alpha \in \mathcal{R}
$$

where $\bar{\phi}_{\alpha}(t) \equiv\left(\phi_{1}+\alpha, \ldots, \phi_{n}+\alpha\right)^{t}$. This property shows that due to its autonomous nature, system (8) remains invariant under time shifts, resulting in a constant phase shift affecting all the oscillator elements. These kinds of systems are more sensitive to numerical noise, which can distort the trajectory of the state variables $\bar{y}(t)$, so it is advisable [31] to use an implicit time-domain integration method, such as Backward-Euler. This improves the numerical stability of the simulated trajectory $\bar{y}(t)$. Using Backward-Euler, system (9) is discretized as.

$$
\frac{\bar{y}\left(t_{k}\right)-\bar{y}\left(t_{k-1}\right)}{t_{k}-t_{k-1}}=\bar{f}\left(\bar{V}\left(t_{k}\right), \bar{\phi}\left(t_{k}\right)\right)
$$

The vector $\bar{y}\left(t_{k}\right)$ of state variables at each $k$-th iteration is calculated by solving system (11) through an optimization method, such as Newton-Raphson. In the simulation, the time steps $\Delta t_{k}=t_{k}-t_{k-1}, k=1, \ldots, P$ must be small enough to capture the dynamic variation of the envelopes $\bar{y}(t)$. A discussion on the initial conditions is given later in this subsection.

Note that using this formulation, the system of $M$ coupled oscillators is modeled with system (11) of $M$ nonlinear complex equations, which reduces noticeably the computational cost when compared with the circuit-level envelope transient system, especially for a high number $M$ of oscillator elements. In contrast with the circuit-level envelope transient technique, system (11) is not subject to uncertainties regarding the start-up of the oscillator elements. This is seen as follows. If the coupling currents entering the $i$-th oscillator is set to zero $\left(\kappa_{i}=\right.$ $0)$, the oscillators operate under free-running conditions, and system (8) simplifies to:

$$
a_{0}^{i}\left(V_{i}\right) V_{i}(t)+a_{1}^{i}\left(V_{i}\right)\left(\dot{V}_{i}(t)+j V_{i}(t) \dot{\phi}_{i}(t)\right)=0
$$


for $i=1, \ldots, M$. Following the same procedure as in [30], a single equation describing the dynamics of the amplitude variable $V_{i}$ (of each oscillator) can derived from each complex equation in (12), obtaining:

$$
\begin{aligned}
& \dot{V}_{i}(t)=g_{i}\left(V_{i}\right), \\
& g_{i}(V)=-\frac{\operatorname{Re} a_{0}^{i}(V) \operatorname{Re} a_{1}^{i}(V)+\operatorname{Im} a_{0}^{i}(V) \operatorname{Im} a_{1}^{i}(V)}{\left|a_{1}^{i}(V)\right|^{2}} V
\end{aligned}
$$

where $R e, I m$ denote real and imaginary parts, respectively. Making use of expression (13), the condition for the dc solution of each $i$-th oscillator to be unstable is $g_{i}^{\prime}(0)>0$, which agrees with the oscillation start-up condition [23], [30]. Since equation (13) is one-dimensional, there always exists an interval $I$ of $V_{i}$ values in the neighborhood of $V_{i}=0$ that belong to the unstable manifold of the dc solution. Then, any initial condition $V_{i}\left(t_{0}=0\right) \in I$ will lead the trajectory of system (11) towards the oscillating solution, provided the time steps $\Delta t_{k}$ take reasonable values. This property remains when introducing each $i$-th oscillator in the coupled system, where the coupling currents $\kappa_{i}$ are activated. This is because, under weak-coupling conditions $\left[Y_{i k}^{c}\left(\omega_{o}\right) \ll Y_{i}\left(0, \omega_{0}\right)\right]$, each $\kappa_{i}$ is a small-amplitude current that, in the neighborhood of the dc solution $\left(V_{i} \simeq 0, i=\right.$ $1, \ldots, M)$, behaves as a second order perturbation, as derived from (3).

\section{B. Application examples}

The new analysis method will be illustrated through its application to two different coupled-oscillator systems. One is based on simple Van der Pol oscillators, which will facilitate the comparison with circuit-level envelope-transient simulations. The other is composed of FET-based oscillators, and the results will be compared with experimental measurements.

\section{1) Van der Pol oscillators}

Initially, the system composed of $M=3$ coupled oscillators of the Van der Pol type, shown in Fig. 2, will be considered. The schematic of each oscillator is shown in Fig. 3. It is composed of an RLC resonator in parallel with a nonlinear voltage-controlled current source, exhibiting negative resistance in small signal. The oscillators are coupled through resistively loaded microstrip lines (width $=0.28 \mathrm{~mm}$, length $=$ $33.9 \mathrm{~mm}$ ) [32]. The admittance matrix of the $n$-port coupling network is calculated in commercial software, providing the coupling terms $Y_{i k}^{c}\left(\omega_{o}\right)$. Note that since the array is unidimensional, the coupling terms of non-adjacent ports vanish, obtaining $Y_{i k}^{c}\left(\omega_{o}\right)=0$ for $|i-k|>1$. As a result, the equations of each oscillator in (8) and (19) are explicitly coupled to the adjacent ones only.

The free-running frequency of each $i$-th oscillator can be tuned by means of the capacitor $C_{i}$. Two qualitatively different cases have been considered. In the first case, the capacitances of the three oscillators are set to the same value: $C_{i}=10 \mathrm{pF}$, $i=1,2,3$, corresponding to the free-running frequency $\omega_{o} \approx$ $2 \pi \cdot 1.6 \mathrm{GHz}$. Therefore, the functions $a_{0}^{i}\left(V_{i}\right)$ and $a_{1}^{i}\left(V_{i}\right)$ are identical for the three oscillators. These functions have been identified through the $\mathrm{HB}$ simulation of a single oscillator isolated from the system, following the procedure described in
[30]. Then, the transient trajectories of the amplitude and phase variables of each oscillator have been calculated by integrating system (9). The initial conditions for the amplitude and phase variables have been respectively set near zero $\left(V_{i}=\right.$ $0.01 V, i=1,2,3)$, and zero $\left(\phi_{i}=0, i=1,2,3\right)$. The simulation results are shown in Fig. 4 . As can be seen, the amplitude and phase trajectories go through a transient state of duration $t_{s} \approx 30$ ns. Once the steady state is reached, the amplitude components become constant, whereas the time variation of the phase components exhibit the same constant slope $\dot{\phi}_{1}=\dot{\phi}_{2}=\dot{\phi}_{3}=\Delta \omega$, providing a common instantaneous frequency $\omega_{o}+\Delta \omega$. This scenario corresponds to a locked solution at the frequency $\omega_{s}=\omega_{o}+\Delta \omega$. In the same figures, the simulation of the same coupled system through circuit-level envelope transient with 7 harmonics in commercial software has been superimposed, with very good agreement.

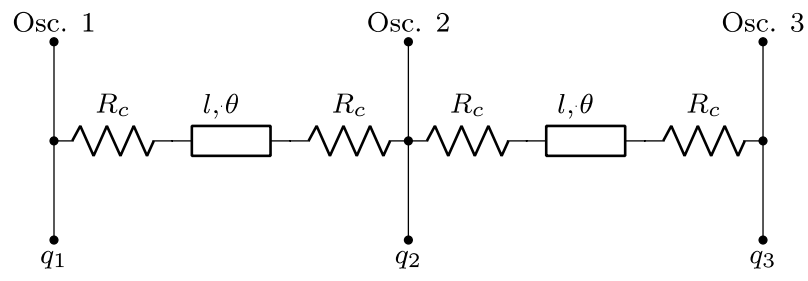

Fig. 2. Schematic of the system of $M=3$ coupled oscillators. The oscillators are coupled through resistively loaded microstrip lines (width $=0.28 \mathrm{~mm}$, length=33.9 mm)

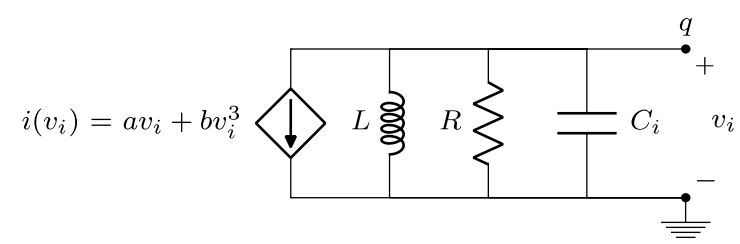

Fig. 3. Schematic of the Van der Pol oscillator. The circuit parameter values are $a=-0.03 \Omega^{-1}, b=0.01 \mathrm{~A} / \mathrm{V}^{3}, L=1 \mathrm{nH}, R=50 \Omega$.

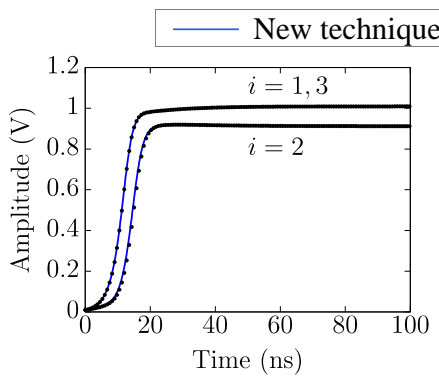

(a)

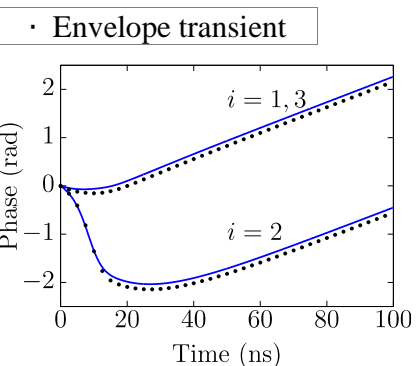

(b)
Fig. 4. System of three coupled Van der Pol oscillators. Locked case, with $C_{i}=$ $10 \mathrm{pF}, i=1,2,3$. (a) Amplitude trajectories. (b) Phase trajectories

In the second case, the capacitances of the three oscillators have been set to $C_{1,3}=11 \mathrm{pF}, C_{2}=10 \mathrm{pF}$. This is a scenario in which the admittance functions $Y_{i}\left(V_{i}, \omega\right)(i=1,2,3)$ are not equal. In this case, as can be seen in Fig. 5(b), the instantaneous frequencies of the outermost oscillators $\omega_{o}+\dot{\phi}_{1,3}$ are different from the central one $\omega_{o}+\dot{\phi}_{2}$. Thus, this capacitor selection leads the system to an unlocked state. The different oscillation frequencies existing in the coupled systems make the amplitude and phase variables exhibit an oscillatory behavior [see Fig. 5(a)]. As in the previous case, the results show an excellent 
agreement with those obtained with circuit-level envelope transient, superimposed in the figure.

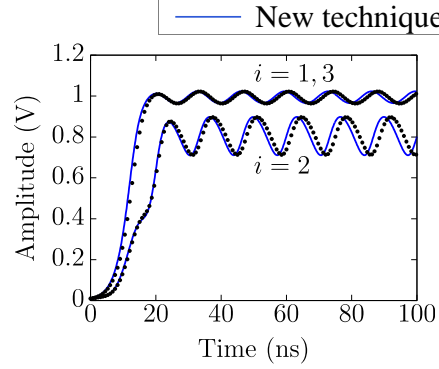

(a)

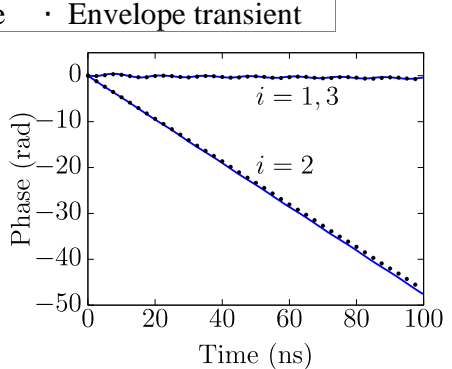

(b)
Fig. 5. System of three coupled Van der Pol oscillators. Unlocked case, with $C_{1,3}=11 \mathrm{pF}, C_{2}=10 \mathrm{pF}$. (a) Amplitude trajectories. (b) Phase trajectories.

\section{2) Transistor-based oscillators}

The formulation has also been applied to a system of three FET-based oscillators that share the same topology, as shown in Fig. 6(a). Each oscillator is designed using a field-effect transistor (NE3210S01) on a RO4003C substrate. A varactor diode (SMV1233) is used as a tuning element with the dc voltage $\eta_{i}, i=1,2,3$. The coupled system is shown in Fig. 6(b)(c). The observation node $q$ of each oscillator agrees with the output node, where a resistance $R_{o}=50 \Omega$ is connected in parallel with the coupling network.

The bivariate nonlinear admittance function is obtained through a double sweep in amplitude and frequency, in the intervals $V_{i} \in\left[10^{-4}, 1.2\right] \mathrm{V}, f \in[5,5.12] \mathrm{GHz}$, The build-up transient to a locked solution of the coupled system is shown in Fig. 7, where it is compared with circuit-level envelopetransient simulations and with experimental measurements. These measurements are carried out connecting the oscillator output signal to a Keysight Infiniium 90804A oscilloscope. The photograph of the measurement set-up is shown in Fig. 6(d). The oscillation start-up is triggered at $t=0$ by an ON/OFF signal applied to the drain bias voltage of the oscillators. As can be seen, there is a good agreement. However, the original sweep in the bivariate function does not allow the efficient prediction of build-up transients and unlocked states with large amplitude and frequency excursions.

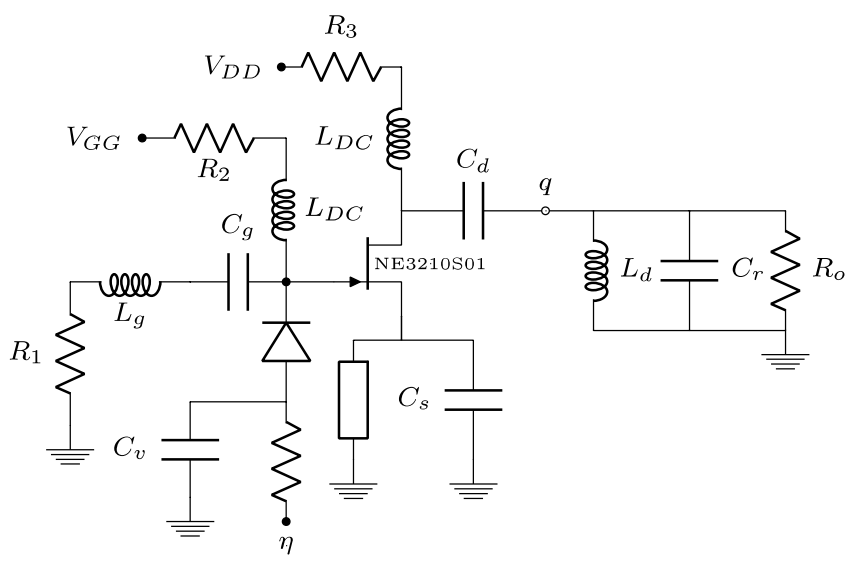

(a)

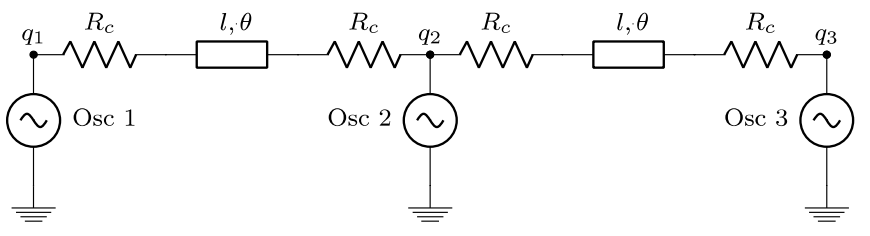

(b)

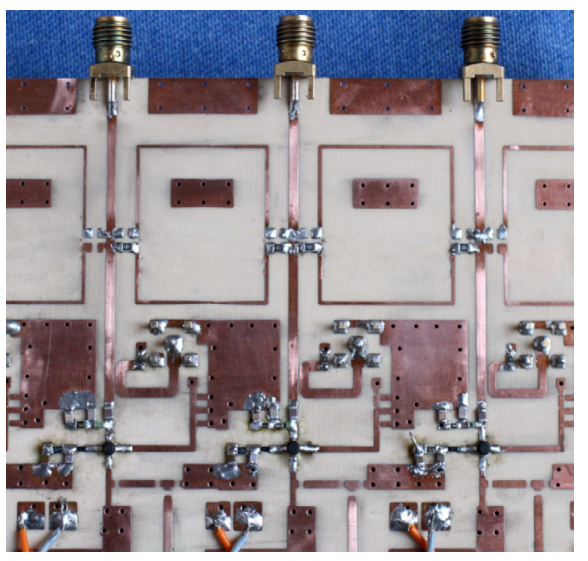

(c)

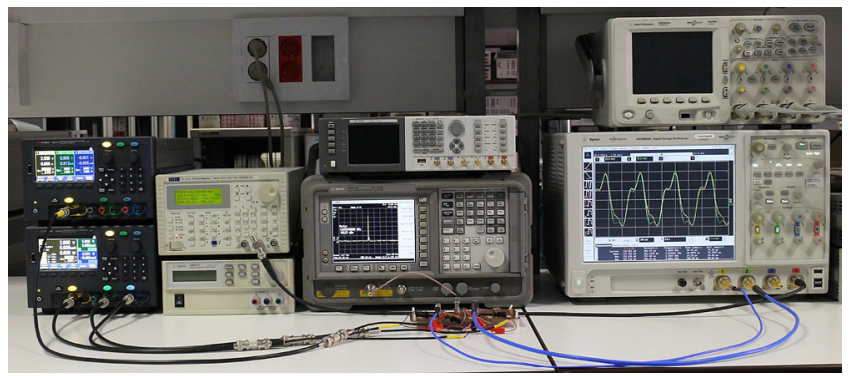

(d)

Fig. 6. Coupled-oscillator system using FET-based oscillators. (a) Schematic of the FET-based oscillator. (b) Schematic of the coupled system. (c) Photograph of implemented system. (d) Measurement set-up.

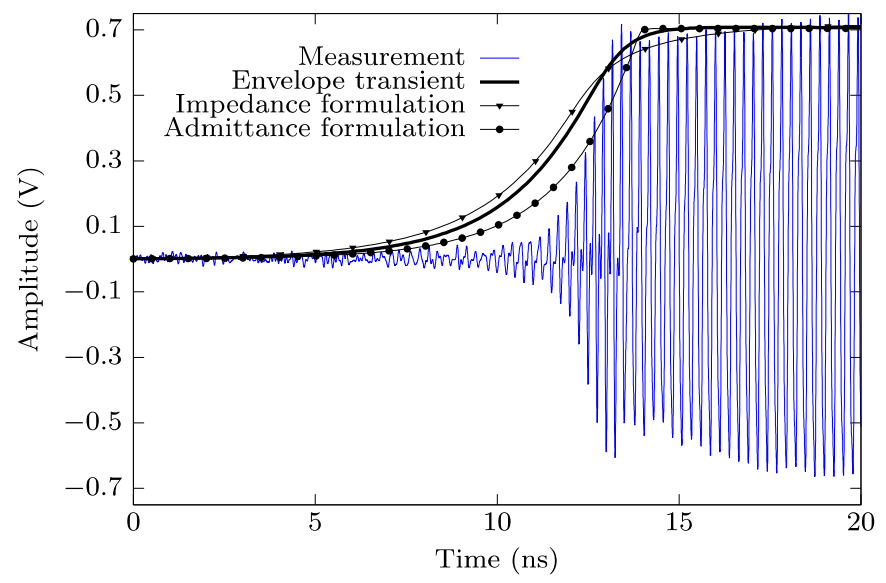

Fig. 7. System of three coupled FET oscillators. Here shown is the measured output voltage of the oscillator $i=1$ when evolving to the locked case, with $\eta_{1,3}=2.095 \mathrm{~V}, \eta_{2}=2 \mathrm{~V}$. The amplitude transient trajectories that are simulated with systems (9) and (20), and with the envelope transient technique, are superimposed. 


\section{ENVELOPE-DOMAIN ANALYSIS BASED ON A NONLINEAR- IMPEDANCE FORMULATION}

\section{A. Motivation}

As will be shown, the impedance-based oscillator models extend the applicability of the nonlinear envelope-domain analysis of the coupled system. As is well known, HB is a numerical method based on error minimization, which may fail to converge in particular circuits. This is often preceded by a stepping procedure, in which the analysis parameter (which may be a simple level coefficient applied to the input sources) undergoes multiple reductions without ever reaching the desired value. The failure is due to the singularity of the Jacobian matrix of the HB system. Geometrically, this singularity implies an infinite slope of the solution curve versus the analysis parameter, which explains why the parameter stepping is unable to cope with the problem [26]-[28]. In inhouse software, it is circumvented through the implementation of complementary continuation methods. One of the most commonly used is parameter switching, based on switching the actual analysis parameter to a fast-varying state variable, and transforming the physical parameter into an unknown to be determined. This parameter switching leads to a structural change in the Jacobian matrix that avoids the singularity.

As in any other HB simulation, the procedure to obtain the nonlinear oscillator models may be subject to convergence problems of the kind described in the previous paragraph. In fact, both the real and imaginary parts of the admittance functions constitute surfaces in the spaces defined by $V, \omega$ and these real and imaginary parts. The singularity of the Jacobian matrix for some pairs of values $V$, $\omega$ would lead to a folding of the surfaces. This can be seen in Fig. 8, where the real and imaginary parts of the admittance function $Y(V, \omega)$ of the oscillator in Fig. 6(a) evaluated at the output node, have been represented versus $V$ and $\omega$.

As can be seen, the admittance function $Y(V, \omega)$ of Fig. 8 is folded and thus multivalued in a small interval of $V$ values. Due to the folding, a double sweep in the parameters $(V, \omega)$ does not provide all the points of the surface, which will prevent the simulation of complex regimes, with relatively large excursions of amplitude and frequency. To circumvent this situation, one can change the excitation signal from the voltage $V$, used to extract the admittance-type model, to a current $I$, which will provide an impedance-type model $Z(I, \omega)$. This is conceptually equivalent to the parameter switching used in in-house HB. Thus, the goal has been to develop an alternative impedancebased formulation, which should be able to increase the scope of application of the new methodology.

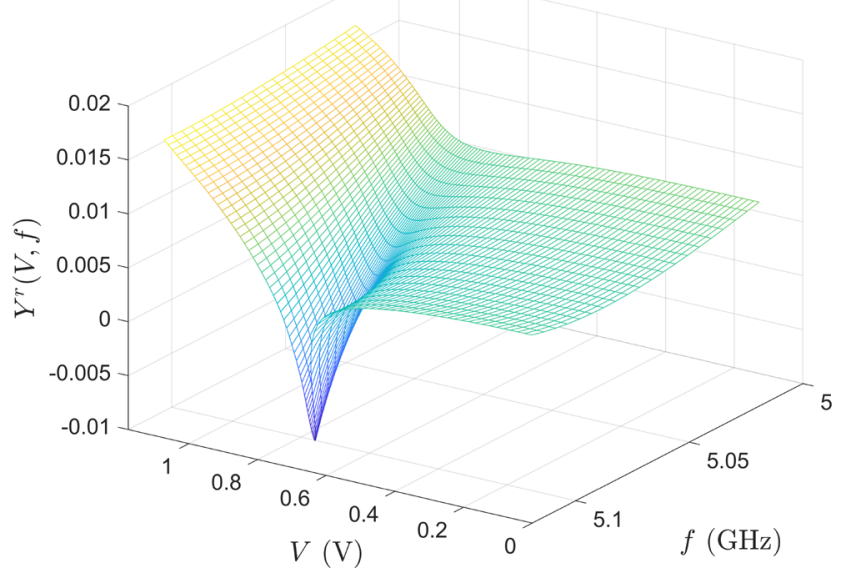

(a)

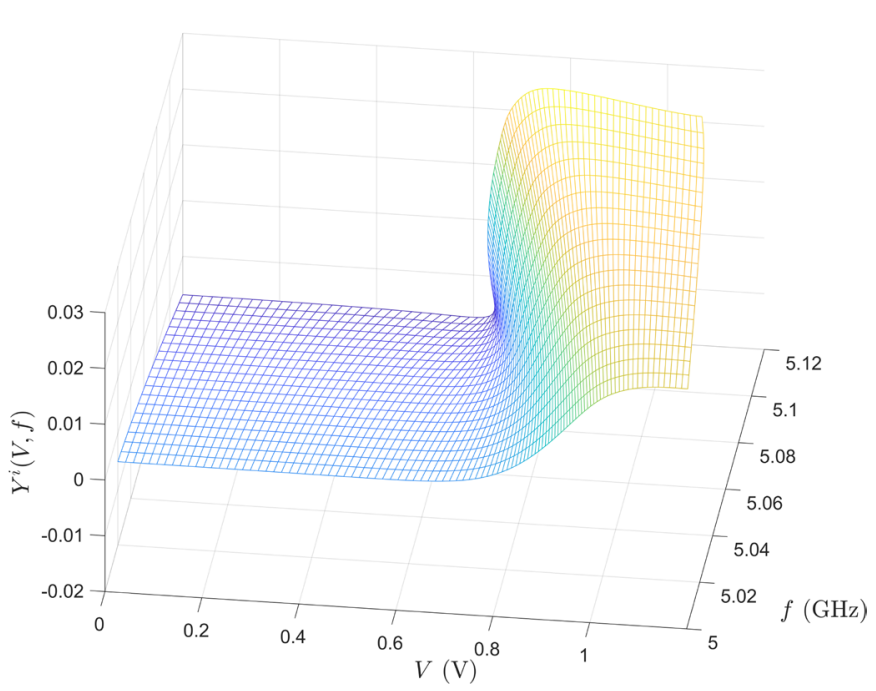

(b)

Fig. 8. Real (a) and imaginary (b) parts of the function $Y(V, f)$, with $f=\omega / 2 \pi$. There exists a small interval of $V$ values inside [0.6,0.8] V where $Y(V, f)$ is multivalued. The varactor diode tuning voltage is $\eta=2 \mathrm{~V}$.

\section{B. Impedance-based formulation}

In the impedance-based formulation, each oscillator is modelled in terms of a nonlinear impedance function $Z_{N}$ containing the active device, in series with a linear impedance function $Z_{L}(\omega)$, as shown in Fig. 9. The partitioning into $Z_{N}$ and $Z_{L}(\omega)$ is carried out taking as a reference the node where the oscillator will be connected to the coupled system. As will be shown in the next section, the passive linear impedance function $Z_{L}(\omega)$, usually corresponding to the oscillator load or a simple resonant circuit, can be easily calculated through an AC simulation. The HB system describing each single oscillator can be written as:

$$
\begin{gathered}
Z\left(\bar{A}^{\prime}, I, \omega\right) I e^{j \varphi}=0, \\
\bar{H}^{\prime}\left(\bar{A}^{\prime}, I, \varphi, \omega\right)=0
\end{gathered}
$$

where $Z\left(\overline{A^{\prime}}, I, \omega\right) \equiv Z_{N}\left(\overline{A^{\prime}}, I, \omega\right)+Z_{L}(\omega)$ will be called the total impedance function. Equation (14a) is the first harmonic 
equation of the Kirchhoff voltage law (KVL) at the closed branch and system (10b) contains the rest of the HB equations. The variables $(I, \varphi)$ agree with the amplitude and phase of the first harmonic component of the current through the closed branch, whereas the vector $\bar{A}^{\prime}$ contains the rest of the harmonics of the state variables. Applying the implicit function theorem, the components in $\overline{A^{\prime}}$ can be derived from equation (14b) in terms of $(I, \varphi, \omega)$ and then introduced in (14a), obtaining the equation $\left[Z_{N}(I, \omega)+Z_{L}(\omega)\right] I e^{j \varphi}=Z(I, \omega) I e^{j \varphi}=0$ that models the free-running oscillator.

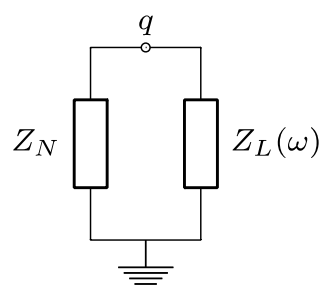

Fig. 9. Schematic of each oscillator in the system when using the impedancebased formulation. The linear impedance function $Z_{L}(\omega)$ corresponding to a passive linear block, can be easily calculated through an AC simulation, usually corresponding to the oscillator load or a simple resonant circuit.

The total impedance function $Z(I, \omega)$ can be calculated using circuit-level HB following the procedure schematized in Fig. 10. A two-terminal network is introduced between the two parts of the oscillator circuit. This network is composed of a singletone current source with amplitude $I_{A G}$, phase zero and frequency $\omega_{A G}$, in parallel with an ideal band stop filter of impedance $Z_{f}(\omega)=\delta\left(\omega-\omega_{A G}\right)$. Then, the circuit of Fig. 10 is simulated in circuit-level $\mathrm{HB}$, applying a double sweep in the amplitude $I_{A G}$ and frequency $\omega_{A G}$, and extracting the total impedance function as:

$$
Z(I, \omega)=\frac{V_{A G}\left(I_{A G}=I, \omega_{A G}=\omega\right)}{I}
$$

where $V_{A G}$ is the first harmonic of the voltage signal $v_{A G}$.

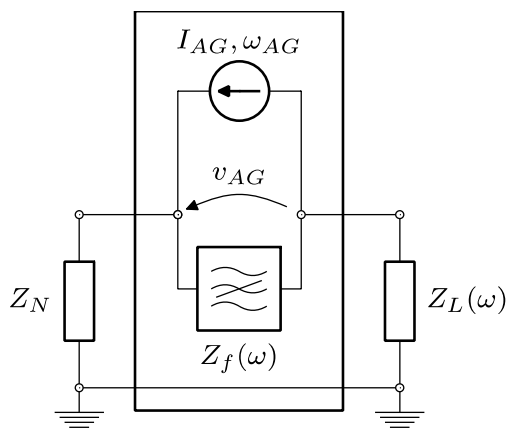

Fig. 10. Schematic of the circuit used to obtain the total impedance function $Z(I, \omega)$.

Once the total impedance function $Z_{i}\left(I_{i}, \omega\right)$ of each $i$-th oscillator has been obtained, it can be used to build the formulation of the coupled system in the following way. The associated block impedances, $Z_{N}^{i}\left(I_{i}, \omega\right)$ and $Z_{L}^{i}(\omega)$, are connected to the node $q_{i}$ of each oscillator. The model of the coupled system will be derived by making use of the variables $\left(U_{i}, X_{i}, \kappa_{i}\right), i=1, \ldots, M$, corresponding to the first harmonic components of the current entering the nonlinear elements $Z_{N}^{i}$, the voltage at the node $q_{i}$, and the current entering the coupling network, respectively.

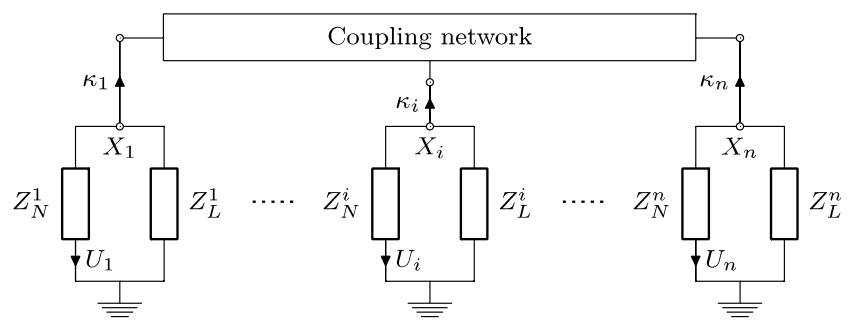

Fig. 11. Schematic of the system of coupled oscillators using the impedancebased formulation.

In the first place, the model of each free-running oscillator will be obtained. According to the schematic of Fig. 11, the KVL equation of each $i$-th oscillator is given by:

$Z_{N}^{i} U_{i}+Z_{L}^{i}(\omega)\left(U_{i}+\kappa_{i}\right)=Z_{i}\left(I_{i}, \omega\right) U_{i}+Z_{L}^{i}(\omega) \kappa_{i}=0$

As in the admittance-based formulation, the current phasors entering the coupling network will be expressed in terms of the admittance matrix of the network:

$$
\kappa_{i}=\sum_{k=1}^{n} Y_{i k}^{c} X_{k}=\sum_{k=1}^{n} Y_{i k}^{c} Z_{N}^{k}\left(I_{k}, \omega\right) U_{k}
$$

Now, introducing (17) in (16), the following system of frequency-domain equations is obtained:

$$
\begin{gathered}
Z_{i}\left(I_{i}, \omega\right) U_{i}+\sum_{k=1}^{n} C_{i}^{k}\left(I_{k}, \omega\right) U_{k}=0 \\
C_{i}^{k}\left(I_{k}, \omega\right) \equiv Y_{i k}^{c} Z_{L}^{i}(\omega)\left(Z_{i}\left(I_{k}, \omega\right)-Z_{L}^{i}(\omega) .\right)
\end{gathered}
$$

with $i=1, \ldots, M$. Equations (18) model the system of coupled oscillators in terms of the current phasors $\bar{U}=\left(U_{1}, \ldots, U_{M}\right)$. The system of equations governing the system dynamics during the transient state can be obtained from (18) applying the procedure followed in (3)-(6), obtaining:

$$
\begin{gathered}
Z_{i}\left(I_{i}(t), \omega_{0}\right) U_{i}-j \frac{\partial Z_{i}\left(I_{i}(t), \omega_{0}\right)}{\partial \omega} \dot{U}_{i}+ \\
+\sum_{k=1}^{n}\left[C_{i}^{k}\left(I_{k}(t), \omega_{0}\right) U_{k}-j \frac{\partial C_{i}^{k}\left(I_{k}(t), \omega_{0}\right)}{\partial \omega} \dot{U}_{k}\right]=0
\end{gathered}
$$

where $i=1, \ldots, M$ and, as in the admittance-based formulation, $\omega_{o}$ is the mean value of the free-running frequencies of the oscillators in the array. Splitting each complex equation in (19) into real and imaginary parts, the whole system can be rewritten in compact form as an autonomous system:

$$
\dot{\bar{U}}(t)=\bar{g}(\bar{U}(t)), \quad \bar{u} \equiv\left(\begin{array}{c}
\bar{U}^{r} \\
\bar{U}^{i}
\end{array}\right)
$$

where $\bar{U}^{r}$ and $\bar{U}^{i}$ are the vectors containing the real and imaginary parts of the current phasors in $\bar{U}(t)$.

The procedure to obtain system (20) is summarized as: 
1) Obtain the total impedance functions $Z_{i}\left(I_{i}, \omega\right)$ of each $i$-th oscillator in circuit-level HB using the two-terminal network of Fig. 10.

2) Calculate the functions $Z_{L}^{i}(\omega)$ of each $i$-th oscillator through an AC simulation.

3) Build nonlinear system of ODEs (20) from these functions and use it to simulate the transient trajectories of the state variables $\bar{U}(t)$ through time integration.

As in the case of the admittance-based formulation, the impedance-based formulation describes the system of $M$ coupled oscillators with a system of $M$ nonlinear complex ODEs, which will be solved in the form (11). This noticeably reduces the computational cost in comparison with the circuitlevel envelope transient, especially in the case of a high number $M$ of oscillator elements. As in the case of the admittance-based model, system (20) is not subject to uncertainties regarding the start-up of the oscillator elements, for the same reasons provided at the end of Section II-A.

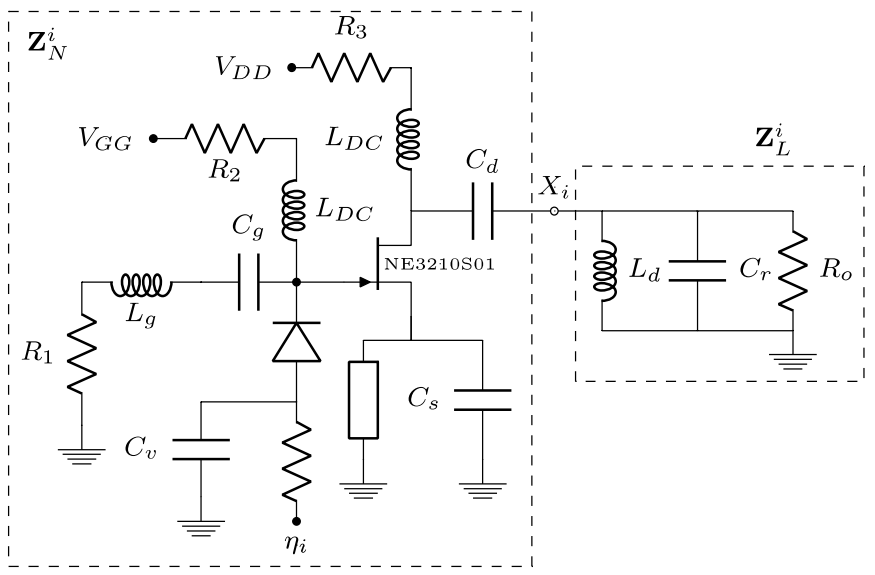

Fig. 12. Schematic of the FET-based oscillator, showing the $Z_{L}$ and $Z_{N}$ blocks.

\section{Application to the system of three FET-based coupled oscillators.}

To apply the impedance-based formulation to the oscillator in Fig. 6(a), this circuit is divided into two parts as shown in Fig. 12: The nonlinear part that contains the active device, providing the impedance function $Z_{N}^{i}\left(I_{i}, \omega\right)$, and the linear part, providing the impedance $Z_{L}^{i}(\omega)$. All the oscillators contain the same linear impedance function $Z_{L}(\omega) \equiv Z_{L}^{i}(\omega)$, whereas the nonlinear impedances can be different, depending on the tuning and bias voltages. In the present analysis, the tuning voltage $\eta_{i}$ has been taken as a parameter. Then, the nonlinear impedance function of each $i$-th oscillator will be denoted as $Z_{N}^{i}\left(I_{i}, \omega\right)=$ $Z_{N}\left(I_{i}, \omega, \eta_{i}\right)$, where the function itself $Z_{N}(I, \omega, \eta)$ is common to the three oscillators. The total impedance function of each oscillator is then given by a common function that will be expressed as:

$$
Z_{i}=Z\left(I_{i}, \omega, \eta_{i}\right)=Z_{L}(\omega)+Z_{N}\left(I_{i}, \omega, \eta_{i}\right)
$$

This function is built by obtaining the function $Z(I, \omega)$ through the procedure described in Section II-B for a collection of $P$ values of $\eta$. Then, function (21) is evaluated through linear interpolation in the set of $\eta$ values. In this subsection, in order to provide a formulation able to simulate the system behavior under small variations of the tuning voltages $\eta_{i}$, a collection of
$P=2$ tuning values $\left(\eta_{0}-\Delta \eta, \eta_{0}+\Delta \eta\right)$, with $\eta_{0}=2 \mathrm{~V}$ and $\Delta \eta=0.1 \mathrm{~V}$, has been chosen. This provides a linear dependence of the function $Z\left(I_{i}, \omega, \eta_{i}\right)$ on the tuning voltage $\eta_{i}$ :

$$
\begin{aligned}
& Z\left(I_{i}, \omega, \eta_{i}\right)=Z\left(I_{i}, \omega, \eta_{0}\right)+ \\
& \quad+\frac{Z\left(I_{i}, \omega, \eta_{0}+\Delta \eta\right)-Z\left(I_{i}, \omega, \eta_{0}-\Delta \eta\right)}{2 \Delta \eta}\left(\eta_{i}-\eta_{0}\right)
\end{aligned}
$$

To show the regular behavior of the impedance surface, Fig. 13 provides the real and imaginary parts of the total impedance function $Z(I, \omega)$ for the same tuning voltage $\eta=2 \mathrm{~V}$ as in the analysis of Fig. 8. As can be seen, the use of the impedancebased formulation avoids the multivalued behavior in all the expected excursion of the current amplitude during its transient dynamics.

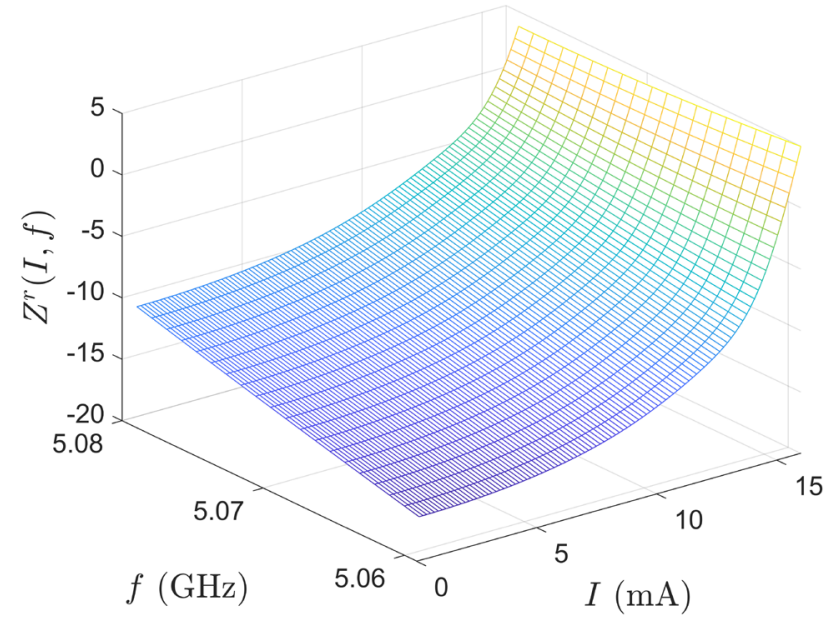

(a)

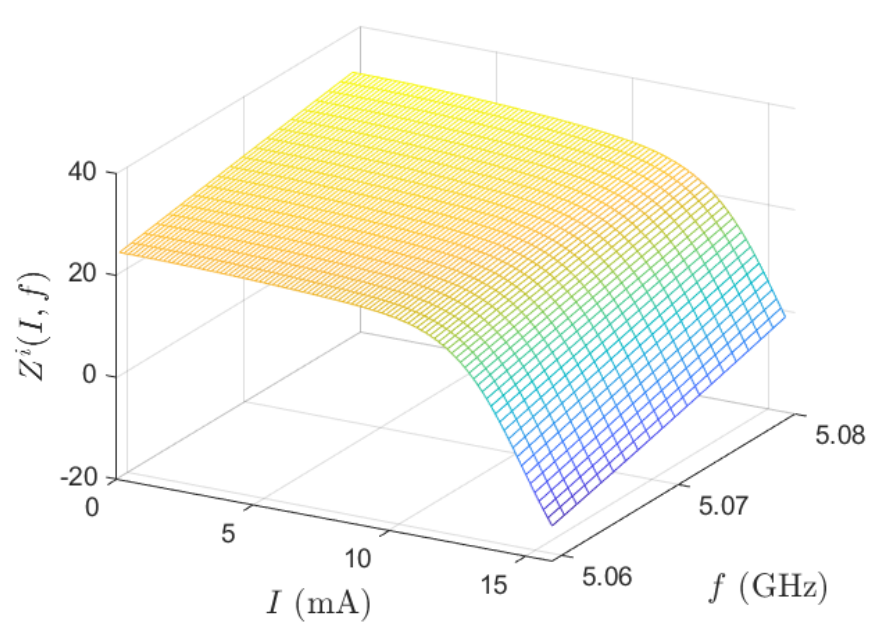

(b)

Fig. 13. Real (a) and imaginary (b) parts of the function $Z(I, f)$, with $f=$ $\omega / 2 \pi$. The use of the impedance-based formulation avoids the multivalued behavior in all the range of current amplitude values used in the analysis. The varactor diode tuning voltage is $\eta=2 \mathrm{~V}$.

In the first analysis, the tuning values $\eta_{1,3}=2.1 \mathrm{~V}, \eta_{2}=2$ $\mathrm{V}$ have been used. The initial conditions for the current variables of system (20) have been set to $U_{i}^{r}=U_{i}^{i}=0.1 \mathrm{~mA}$, 
for $i=1,2,3$. The fundamental frequency used in the simulation of system (19) is $f_{0}=5.07 \mathrm{GHz}$, given by the mean value of the free-running frequencies of the three oscillators. The transient evolution to the steady state of the amplitudes $I_{i}(t)$ of the current phasors, calculated through system (20), are shown in Fig. 14. As can be seen, the system evolves towards a locked state after a transient of duration $t_{s} \approx 14 \mathrm{~ns}$, where the amplitude variables become constant. As in the previous analysis, the results have been validated with the envelope transient technique.

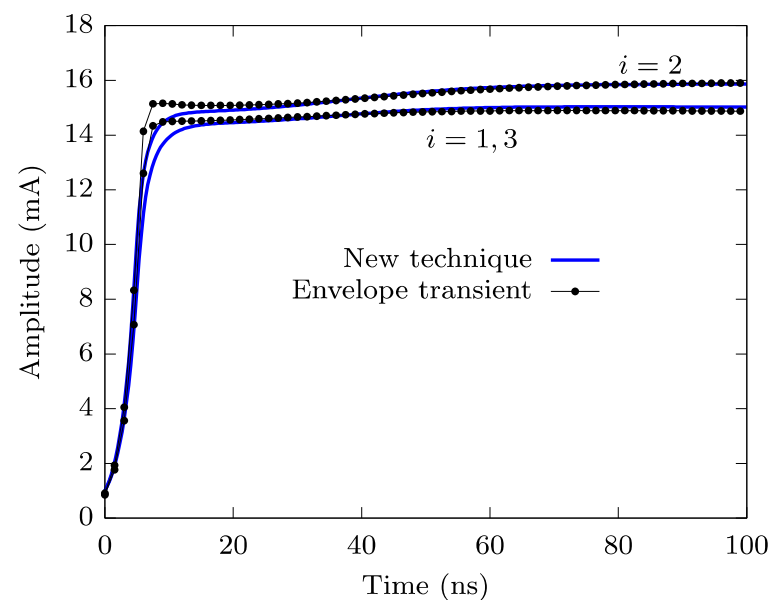

Fig. 14. System of three coupled FET oscillators: transient to the locked state. Simulated amplitude trajectories for the locked case, with $\eta_{1,3}=2.1 \mathrm{~V}, \eta_{2}=2$ $\mathrm{V}$. The results of the envelope-transient simulation are superimposed.

In the second analysis, the three tuning voltages have been equated to the value $\eta_{1,2,3}=2 \mathrm{~V}$. The simulation results are shown in Fig. 15, where an unlocked state is observed. The transient duration is similar to the one in the locked case, and both the new technique and the envelope transient show a similar oscillatory behavior. Note that, in this case, each oscillator exhibits a different oscillation frequency, yielding a beat frequency that constitutes a system unknown. The small discrepancies in these beat frequencies give rise to a slightly larger discrepancy between the semi-analytical formulation and the circuit level simulation than in the case of the injectionlocked regime in Fig. 14.

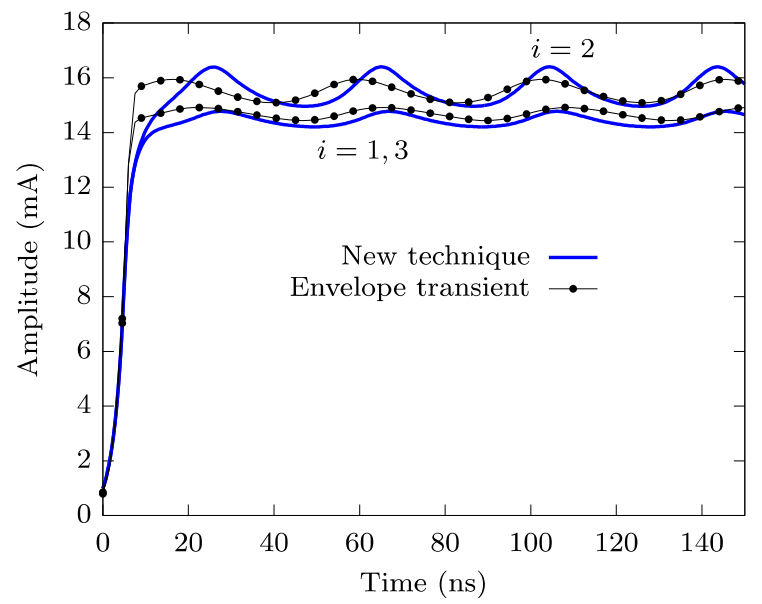

Fig. 15. System of three coupled FET oscillators: transient to the unlocked state. Simulated amplitude trajectories for the unlocked case, with $\eta_{1}=\eta_{2}=$ $\eta_{3}=2 \mathrm{~V}$. The results of the envelope-transient simulation are superimposed.

Finally, the transient trajectory of the amplitude of the oscillator $i=1$ has been measured when evolving to the locked case of Fig. 7. Since the oscilloscope provides the output voltage signals, these variables have been extracted from the simulation results in the following way. Once system (20) has been solved through numerical integration, the output voltage phasor of each $i$-th oscillator can be calculated from the current phasor $U_{i}(\mathrm{t})$ as:

$$
\begin{aligned}
X_{i}(t)=Z_{N}^{i}\left(I_{i}(t), \omega_{0}, \eta_{i}\right) U_{i}(t) & \\
& -j \frac{\partial Z_{N}^{i}\left(I_{i}(t), \omega, \eta_{0}\right)}{\partial \omega} \dot{U}_{i}(t),
\end{aligned}
$$

where $Z_{N}^{i}\left(I_{i}(t), \omega_{0}, \eta_{i}\right)=Z_{i}\left(I_{i}(t), \omega_{0}, \eta_{i}\right)-Z_{L}^{i}\left(\omega_{0}\right)$. The measured transient to the locked state is compared in Fig. 7 with the amplitude $V_{1}(t)=\left|X_{1}(t)\right|$ resulting from the simulation of system (20) and extracted from (23), and the envelope transient results. The results obtained with the admittance-based model have been superimposed, usable in this case because of the smaller excursion of amplitudes when evolving to a locked solution.

\section{NONLINEAR DYNAMICS OF THE SYSTEM OF THREE FET- BASED COUPLED OSCILLATORS}

The new technique allows the study of the influence of any circuit parameter on the coupled system dynamics. To illustrate this point, in the next two subsections, the evolution of the steady-state solution as the tuning voltages $\eta_{i}$ are varied will be analyzed. After that, in a third subsection, the ability of the new formulation to predict the system response to input modulated signals will be tested.

\section{A. Bifurcation map}

In this analysis, the tuning voltages of the outermost oscillators have been expressed as $\eta_{1,3}=2.1 \mathrm{~V}+\Delta \eta$, where $\Delta \eta$ is a detuning voltage that has been swept in the range $[-25,20]$ $\mathrm{mV}$. The tuning voltage of the central oscillator has been fixed to $\eta_{2}=2 \mathrm{~V}$. In Fig. 16, the magnitude of the time varying current amplitude of the central oscillator $I_{2}(t)$ has been represented versus the parameter $\Delta \eta$. The initial transient to the steady state solution has been removed from each simulation. Then, from the projection of $I_{2}(t)$ (or any other state variable) versus $\Delta \eta$, one can infer the system bifurcation behavior. Locked states should provide a constant value of the current magnitude, which in the projection of Fig. 16 corresponds to a single point. In contrast, unlocked states should provide a vertical point distribution, corresponding to the projection of the time-varying magnitude.

The locked state simulation of Fig. 14 corresponds to the value $\Delta \eta=0$ and as can be seen in the central region about this value, a single point is obtained for each $\Delta \eta$, indicating that this envelope has become constant and a locked state has been attained. As the detuning parameter $\Delta \eta$ is moved away from this central value, a local/global bifurcation takes place, which gives rise to the onset of an autonomous quasi-periodic solution, 
exhibiting a zero value of the beat frequency at the bifurcation point. To the best of our knowledge, this is the first time that this kind of bifurcation map is obtained in a realistic coupledoscillator system, described with reduced order-models of its transistor-based oscillator elements. The system behavior in the vicinity of the bifurcation points will be analyzed in the next subsection.

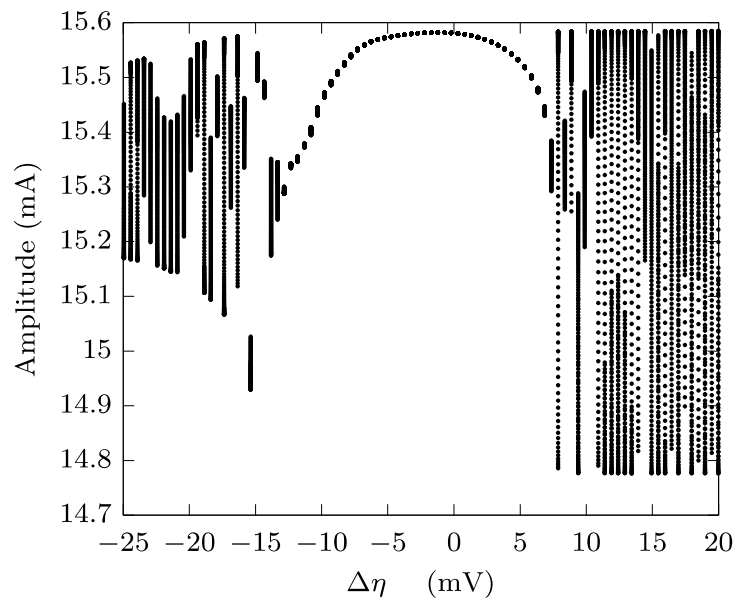

Fig. 16. System of three coupled FET oscillators: bifurcation map. The tuning voltages of the outermost oscillators have been expressed as $\eta_{1,3}=2.1 \mathrm{~V}+\Delta \eta$, where $\Delta \eta$ is a detuning voltage that has been swept in the range $[-25,20] \mathrm{mV}$. The tuning voltage of the central oscillator has been fixed to $\eta_{2}=2 \mathrm{~V}$. For each $\Delta \eta$ value, the resulting time varying current amplitude of the central oscillator $I_{2}(t)$ has been represented

\section{B. Near-locking behavior}

Near the locking conditions $(\Delta \eta \approx 7 \mathrm{mV})$, the transient trajectories exhibit the behavior shown in Fig. 17. There is a long transient during which the current amplitudes of the three oscillators seem to keep constant (which would indicate a locked behavior). However, after a long time $t_{u}$, the current amplitudes reach an oscillatory steady state of low frequency, so the coupled system is actually unlocked. It is observed that the time-varying current amplitudes of the outermost oscillators $i=1,3$ oscillate in quadrature, whereas in the unlocked state simulated in Fig. 15 for a much higher detuning $(\Delta \eta \approx-100$ $\mathrm{mV}$ ), these components oscillate in phase. The results of the impedance-based formulation have been compared with those of the circuit-level envelope transient. As can be seen, both simulations predict the same phenomenon. The difference in the value of $t_{u}$ is due to the fact that this value is extremely dependent on the initial conditions. These conditions are not exactly the same in the two simulations, since system (20) is of sixth order, whereas the order of the circuit-level envelope transient system is much higher. Again, to the best of our knowledge, this is also the first time that a realistic coupledoscillator system is simulated in near locked conditions using reduced-order models of its transistor-based oscillator elements.

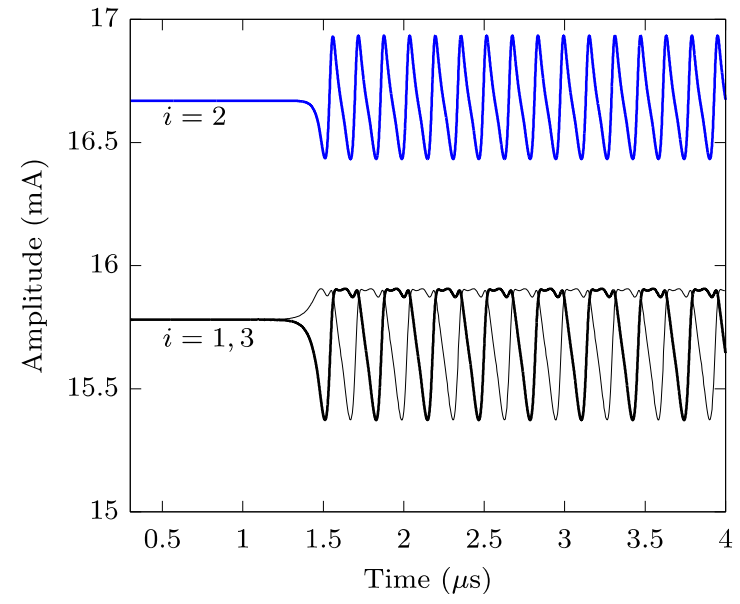

(a)

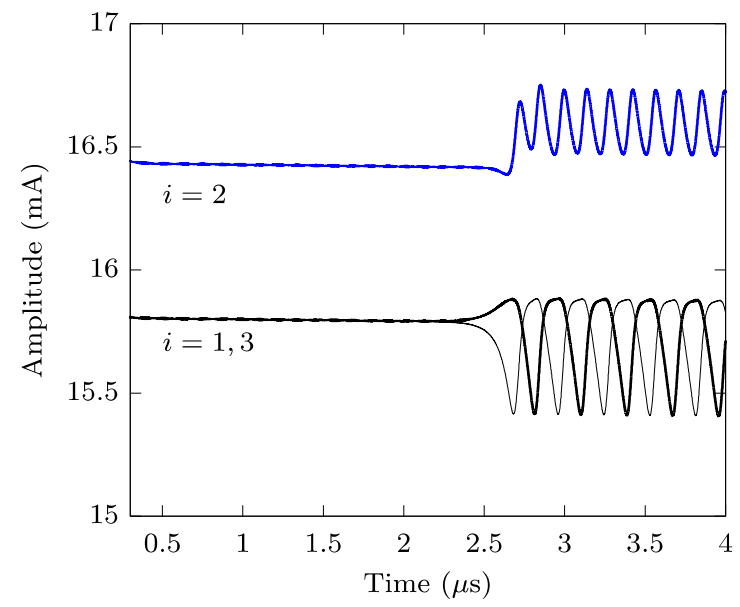

(b)

Fig. 17. System of three coupled FET oscillators: simulated amplitude trajectories for the near-locking case, with $\eta_{1}=\eta_{3}=2.107 \mathrm{~V}$ and $\eta_{2}=2 \mathrm{~V}$. (a) Impedance-based formulation. (b) Circuit-level envelope transient simulation.

In order to obtain the spectrum corresponding to the quasilocked state of Fig. 17, first, the first harmonic voltage signals $X_{i}(t)$ at each oscillator output node have been calculated through (23). Then, the Fourier transform $X_{i}(f)$ of each timevarying harmonic component $X_{i}(t)$ is obtained. Finally, the power spectrum is calculated as $p_{i}(f)=\left|X_{i}(f)\right|^{2} / R_{o}$, with $R_{o}=50 \Omega$. Note that the variable $f$ is the frequency offset from the fundamental frequency $f_{0}$ used in the simulation of system (19). In Fig. 18(a), the power spectrum $p_{1}(f)$ is shown. The measured output power spectrum under the same appears in Fig. 18(b). As can be seen, both spectra show a similar distribution of maxima, corresponding to a near-locking state [33]. 


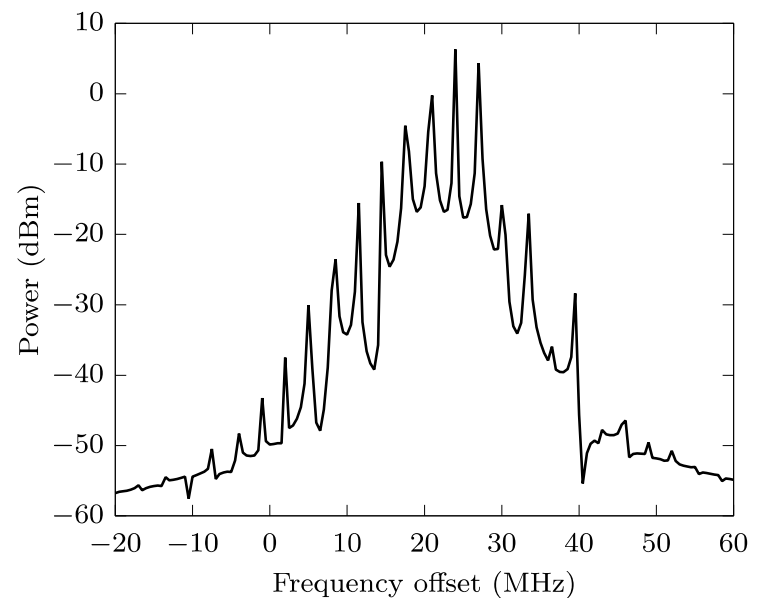

(a)

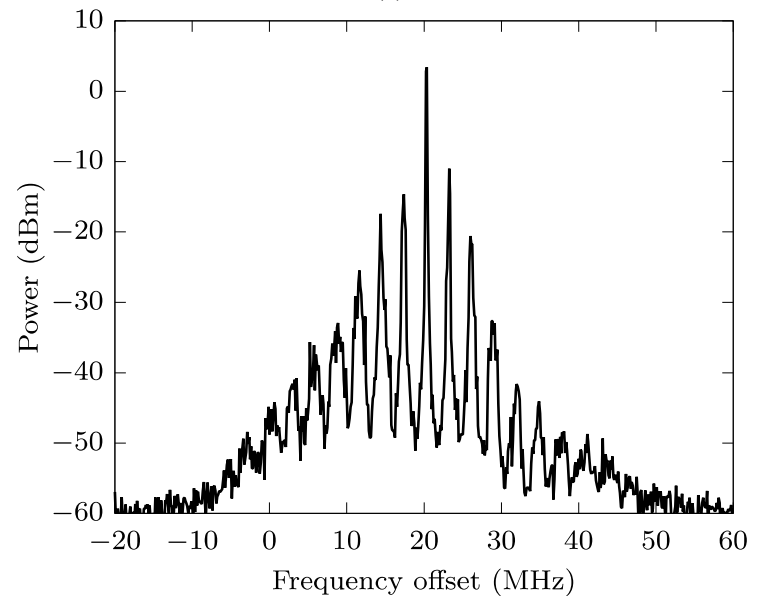

(b)

Fig. 18. System of three coupled FET oscillators: spectrum of the output signal of the oscillator $i=1$ in the near-locking state of Fig. 17. (a) Spectrum simulated with the new technique. (b) Measured spectrum.

\section{Operation under oscillator bias modulation}

In this subsection, the ability of the new formulation to predict the system response to input modulated signals has been tested. For this purpose, the drain bias voltage $V_{D D}^{i}$ of each $i$-th oscillator has been included as a parameter following the same procedure as in (21), obtaining a common total impedance function:

$$
Z_{i}=Z\left(I_{i}, \omega, \eta_{i}, V_{D D}^{i}\right)=Z_{L}(\omega)+Z_{N}\left(I_{i}, \omega, \eta_{i}, V_{D D}^{i}\right)
$$

The system of coupled oscillators has been simulated when the drain bias voltage $V_{D D}^{2}(t)$ of the middle oscillator is a pulseshaped signal. In this case, the total impedance function $Z_{2}$ of that oscillator must be updated for each time value in system (20) according to the drain bias value $V_{D D}^{2}(t)$. To carry out this simulation, the impedance function (24) has been calculated in circuit-level HB in a grid $(P \times Q)$ composed of $P=2$ tuning values $\left(\eta_{0}-\Delta \eta, \eta_{0}+\Delta \eta\right)$ with $\eta_{0}=2 \mathrm{~V}$ and $\Delta \eta=0.1 \mathrm{~V}$, and $Q=50$ drain bias values, which are equispaced in the range $V_{D D} \in[0.9,1.6] \mathrm{V}$.

In the simulation, the pulsed-shaped bias voltage $V_{D D}^{2}(t)$, takes the values $1.4 \mathrm{~V}$ and $1 \mathrm{~V}$, whereas the outermost bias voltages are fixed to $V_{D D}^{1,3}=1.4 \mathrm{~V}$. The initial conditions for the current variables of system (20) have been set to $U_{i}^{r}=U_{i}^{i}=$
$0.1 \mathrm{~mA}$, for $i=1,2,3$. The transient evolution to the steady state of the amplitudes $I_{i}(t)$ of the current phasors has been calculated through system (20). Then, these simulation results have been introduced in (23) to calculate the first harmonic voltage signals $X_{i}(t)$ at each oscillator output node. The magnitudes $V_{i}(t)=\left|X_{i}(t)\right|$ of these signals are shown in Fig. 19(a). The results of the envelope-transient simulation are superimposed.

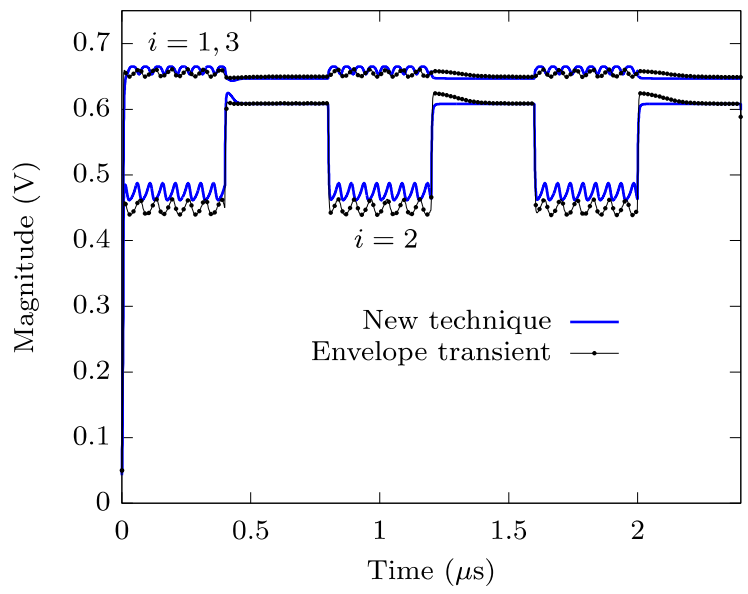

(a)

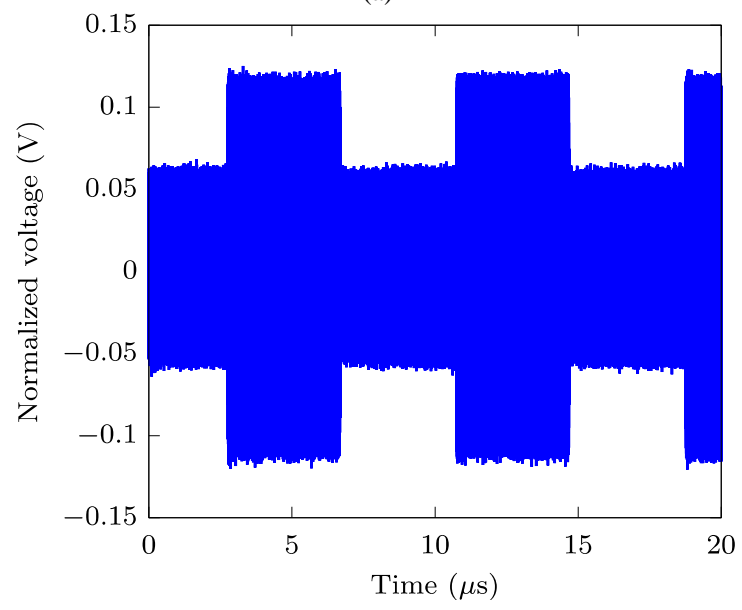

(b)

Fig. 19. System of three coupled FET oscillators. (a) Simulated amplitude trajectories under oscillator bias modulation, with $\eta_{1}=\eta_{3}=2.1 \mathrm{~V}$ and $\eta_{2}=2$ $\mathrm{V}$. The drain bias voltage of the middle oscillator is a pulsed-modulated signal $V_{D D}^{2}(t)$ taking the values $1.4 \mathrm{~V}$ and $1 \mathrm{~V}$, whereas the outermost bias voltages are fixed to $V_{D D}^{1,3}=1.4 \mathrm{~V}$. The results of the envelope-transient simulation are superimposed. (b) Measured output voltage $v_{2}(t)$ of the middle oscillator, showing the same qualitative behavior as the simulation results.

In order to show the capabilities of the technique, the initial transient to the modulated state has also been shown in the figure, which is in agreement with the envelope transient simulation. As can be seen, the system evolves alternatively from the unlocked $\left(V_{D D}^{2}=1 \mathrm{~V}\right)$ to the locked state $\left(V_{D D}^{2}=1.4\right.$ $\mathrm{V})$, with good agreement between both techniques. The period of the pulse-shaped bias has been set to $T_{D D}=0.8 \mu \mathrm{s}$ to allow the observation of the different behavior of the magnitudes in the unlocked (oscillating) and locked (non-oscillating) states.

Finally, the coupled system response to a pulsed-shaped bias voltage $V_{D D}^{2}(t)$ has been measured. The time-domain 
output voltage signal $v_{2}(t)$ of the middle oscillator is shown in Fig. 19(b). As can be seen, the amplitude of this signal shows the qualitative behavior predicted by the simulation results as $V_{D D}^{2}(t)$ moves the system between the two states. In the measurement, the period of the pulse-shaped bias is $T_{D D}=$ $8 \mu s$.

\section{CONCLUSION}

A realistic formulation to predict the transient dynamics of coupled-oscillator systems has been presented. The formulation relies on nonlinear reduced-order models of the individual oscillators, extracted from harmonic-balance simulations. To increase the applicability, both nonlinear admittance- and impedance-type models are considered, extracted under a voltage and current excitation, respectively, at the node/branch where the oscillator is connected to the coupled system. The formulation has been illustrated through its application to a system of three FET-based coupled oscillators at $5 \mathrm{GHz}$, and the results have been compared with circuit-level envelopetransient simulation — which will fail or be impractical under a large number of elements - as well as with experimental measurements. The reduced-order envelope-domain system should be able to predict both locked and unlocked states, as well as the build-up transients. This realistic and efficient analysis of the nonlinear dynamics of the coupled system is presented here for the first time, to the best of our knowledge.

\section{REFERENCES}

[1] J. J. Lynch and R. A. York, "Synchronization of oscillators coupled through narrow-band networks," IEEE Trans. Microw. Theory Tech., vol. 49, no. 2, pp. 237-249, Feb. 2001.

[2] T. Heath, "Simultaneous beam steering and null formation with coupled, nonlinear oscillator arrays,” IEEE Trans. Antennas Propag., vol. 53, no. 6, pp. 2031-2035, Jun. 2005.

[3] W. Lai, S. Jang, and C. Wang, "Concurrent VCO using capacitive coupled oscillators," in 2017 2nd IEEE Int. Conf. Integr. Circuits Microsystems, Nanjing, China, 2017, pp. 1-4.

[4] D. Lee et al., "NbO2-Based Frequency Storable Coupled Oscillators for Associative Memory Application,” IEEE J. Electron Devices Soc., vol. 6, no. 1, pp. 250-253, Jan. 2018.

[5] R. J. Pogorzelski, "Continuum modeling of coupled oscillator arrays with coupling delay,” Radio Sci., vol. 43, no. 4, pp. 1-12, Aug. 2008.

[6] R. Moussounda, "Analysis and design of coupled-oscillator arrays for microwave systems," Ph.D. dissertation. Dept. Elect. Comput. The Ohio State Universityv., Columbus, OH, USA, 2013.

[7] T. Kijsanayotin, J. Li, and J. F. Buckwalter, "A 70-GHz LO PhaseShifting Bidirectional Frontend Using Linear Coupled Oscillators," IEEE Trans. Microw. Theory Tech., vol. 65, no. 3, pp. 892-904, Mar. 2017.

[8] L. Qi, Q. Xie, L. Deng, and Z. Wang, "A novel terahertz phased array based on coupled oscillators," in 2018 IEEE MTT-S Int. Wirel. Symp., Chengdu, China, May 2018, pp. 1-3.

[9] Q. Xie, H. Wang, J. Li, and Z. Wang, "Progressive LO phase shifting technique with linear coupled oscillators in 90nm BiCMOS," in 2018 IEEE MTT-S Int. Wirel. Symp. IWS 2018 - Proc., Chengdu, China, May 2018, pp. 1-4.

[10] R. Andrawis and K. Roy, "Antiferroelectric Tunnel Junctions as EnergyEfficient Coupled Oscillators: Modeling, Analysis, and Application to Solving Combinatorial Optimization Problems," IEEE Trans. Electron Devices, vol. 67, no. 7, pp. 2974-2980, Jul. 2020.

[11] Z. Wang and Y. Wang, "Pulse-Coupled Oscillators Resilient to Stealthy Attacks,” IEEE Trans. Signal Process., vol. 66, no. 12, pp. 3086-3099, Jun. 2018.

[12] M. Ponton, S. Hernandez, and A. Suarez, "Phase-sensitivity analysis of injection-locked mutually coupled oscillators,” in Eur. Microw. Week
2017, Nuremberg, Germany, Dec. 2017, pp. 771-774.

[13] A. Suarez, Analysis and Design of Autonomous Microwave Circuits. Hoboken, NJ, USA: Wiley, 2009.

[14] S. Sancho, F. Ramirez, and A. Suarez, "Stochastic analysis of cycle slips in injection-locked oscillators and analog frequency dividers," IEEE Trans. Microw. Theory Tech., vol. 62, no. 12, pp. 3318-3332, Dec. 2014.

[15] A. Suárez, F. Ramírez, and S. Sancho, "Stability and noise analysis of coupled-oscillator systems,” IEEE Trans. Microw. Theory Tech., vol. 59, no. 4 Part 1, pp. 1032-1046, Apr. 2011.

[16] A. Suarez, S. Sancho, and F. Ramirez, "General formulation for the analysis of injection-locked coupled-oscillator systems," IEEE Trans. Microw. Theory Tech., vol. 61, no. 12, pp. 4730-4744, Dec. 2013.

[17] E. Ngoya and R. Larcheveque, "Envelop transient analysis: A new method for the transient and steady state analysis of microwave communication circuits and systems," in IEEE MTT-S Int. Microw. Symp. Dig., San Francisco, CA, USA, Jun. 1996, vol. 3, pp. 1365-1368.

[18] N. B. Carvalho, J. C. Pedro, and W. Jang, "Nonlinear RF circuits and systems simulation when driven by several modulated signals," IEEE Trans. Microw. Theory Tech., vol. 54, no. 2, pp. 572-579, Feb. 2006.

[19] K. S. Kundert, "Introduction to RF Simulation and Its Application," IEEE J. Solid-State Circuits, vol. 34, no. 9, pp. 1298-1319, Sep. 1999.

[20] A. Suarez, F. Ramirez, S. Sancho, and J. M. Collantes, "Global stability analysis of coupled-oscillator systems," IEEE Trans. Microw. Theory Tech., vol. 63, no. 1, pp. 165-180, Jan. 2015.

[21] R. A. York, P. Liao, and J. J. Lynch, "Oscillator Array Dynamics with Broadband N-Port Coupling Networks," IEEE Trans. Microw. Theory Tech., vol. 42, no. 11, pp. 2040-2045, Nov. 1994.

[22] F. Ramírez, A. Suárez, and S. Sancho, "Harmonic-balance technique for the shortening of the initial transient of microwave oscillators," in IEEE MTT-S Int. Microw. Symp. Dig., Long Beach, CA, USA, Jun. 2005, pp. 805-808.

[23] K. Kurokawa, "Some basic characteristics of broadband negative resistance oscillator circuits,” Bell Syst. Tech. J., vol. 48, no. 6, pp. 19371955, Jul. 1969.

[24] S. Sancho, A. Suarez, and F. Ramirez, "Analysis of the Transient Dynamics of Coupled-Oscillator Systems," in 2020 IEEE/MTT-S Int. Microw. Symp., Los Angeles, CA, USA, Aug. 2020, pp. 385-388.

[25] V. Rizzoli and A. Neri, "State of the Art and Present Trends in Nonlinear Microwave CAD Techniques," IEEE Trans. Microw. Theory Tech., vol. 36, no. 2, pp. 343-365, Feb. 1988.

[26] A. Ushida and L. O. Chua, "Tracing solution curves of non-linear equations with sharp turning points," Int. J. Circuit Theory Appl., vol. 12, no. 1, pp. 1-21, Jan. 1984.

[27] D. Hente and R. H. Jansen, "Frequency Domain Continuation Method for the Analysis and Stability Investigation of Nonlinear Microwave Circuits," IEE Proc. H Microwaves, Antennas Propag., vol. 133, no. 5, pp. 351-362, Oct. 1986.

[28] J. S. Roychowdhury and R. C. Melville, "Homotopy techniques for obtaining a DC solution of large-scale MOS circuits," in Proc. 1996 33rd Annu. Des. Autom. Conf., Las Vegas, NV, USA, Jun. 2003, pp. 286-291.

[29] A. Suarez, S. Sancho, and F. Ramirez, "Growth-rate function for the nonlinear analysis of the transient dynamics of microwave oscillators," in IEEE MTT-S Int. Microw. Symp. Dig., San Francisco, CA, USA, May 2016, vol. 2016-August.

[30] S. Sancho, A. Suarez, F. Ramirez, and M. Ponton, "Analysis of the Transient Dynamics of Microwave Oscillators," IEEE Trans. Microw. Theory Tech., vol. 67, no. 9, pp. 3562-3574, Sep. 2019.

[31] T. S. Parker and L. O. Chua, Practical Numerical Algorithms for Chaotic Systems. Springer, New York, NY, 1989.

[32] R. A. York, "Nonlinear Analysis of Phase Relationships in Quasi-Optical Oscillator Arrays," IEEE Trans. Microw. Theory Tech., vol. 41, no. 10, pp. 1799-1809, Oct. 1993.

[33] B. Razavi, "A study of injection locking and pulling in oscillators," IEEE J. Solid-State Circuits, vol. 39, no. 9, pp. 1415-1424, Sep. 2004. 


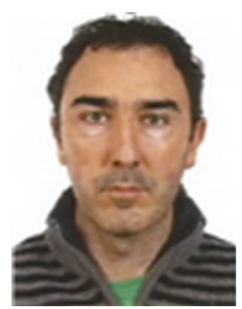

Sergio Sancho (A'04-M’04) received the degree in Physics from Basque Country University in 1997. In 1998 he joined the Communications Engineering Department of the University of Cantabria, Spain, where he received the Ph.D. degree in Electronic Engineering in February 2002. At present, he works at the University of Cantabria, as an Associate Professor of its Communications Engineering Department. His research interests include the nonlinear analysis of microwave autonomous circuits and frequency synthesizers, including stochastic and phase-noise analysis.

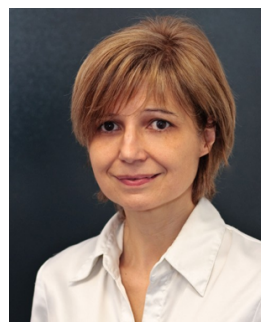

Almudena Suárez (M’96-SM'01-F'12) was born in Santander, Spain. She received the Licentiate degree in Electronic Physics and the Ph.D. degree from the University of Cantabria, Santander, Spain, in 1987 and 1992, respectively, and the Ph.D. degree in Electronics from the University of Limoges, Limoges, France, in 1993. She is currently a Full Professor with the Communications Engineering Department, University of Cantabria. She co-authored Stability Analysis of Nonlinear Microwave Circuits (Artech House, 2003) and authored Analysis and Design of Autonomous Microwave Circuits (IEEE-Wiley, 2009).

Prof. Suárez is an member of the Technical Committees of the IEEE Microwave Theory and Techniques Society (IEEE MTT-S) International Microwave Symposium (IMS) and the European Microwave Conference. She was an IEEE Distinguished Microwave Lecturer from 2006 to 2008. She is a member of the Board of Directors of the European Microwave Association. She was the Coordinator of the Communications and Electronic Technology Area for the Spanish National Evaluation and Foresight Agency between 2009 and 2013. In 2014 and 2015 she was the co-chair of IEEE Topical Conference on RF Power Amplifiers (PAWR). Prof. Suárez is an Associate Editor of the IEEE Transactions on Microwave Theory and Techniques and the IEEE Microwave Magazine.

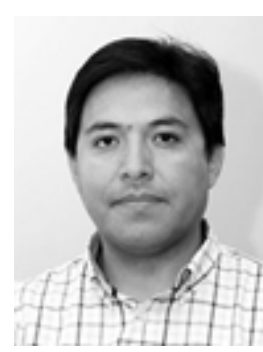

Franco Ramírez (S'03-A'05-M'05-SM'16) obtained the Licentiate degree in electronic systems engineering degree from the Military School of Engineering (EMI) in La Paz, Bolivia, in 2000 and the Ph.D. degree in Communications Engineering from the University of Cantabria, Santander, Spain in 2005. From 1999 to 2000, he worked for Ericsson de Bolivia Telecomunicaciones, where he was involved in projects related with GSM and TDMA technologies. From 2009 to 2013, Dr. Ramírez was a Research Fellow of the "Ramón y Cajal” Programme, funded by the Spanish Ministry of Science and Innovation, at the Communications Engineering Department of the University of Cantabria, where he is currently an Associate Professor. His research interests include phase noise, stability and the development of nonlinear techniques for the analysis and design of autonomous microwave circuits. 